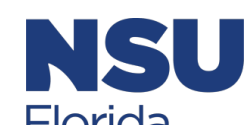

Florida

Spring 2015

\title{
Origins of an Unmarked Georgia Cemetery Using Ancient DNA Analysis
}

Andrew T. Ozga

Raul Y. Tito

Brian M. Kemp

Hugh Matternes

Alexandra J. Obregon-Tito

See next page for additional authors

Follow this and additional works at: https://nsuworks.nova.edu/cnso_bio_facarticles

Part of the Biological and Physical Anthropology Commons, Biology Commons, and the Genetics Commons 
Authors

Andrew T. Ozga, Raul Y. Tito, Brian M. Kemp, Hugh Matternes, Alexandra J. Obregon-Tito, Leslie Neal, and Cecil M. Lewis Jr. 
Origins of an Unmarked Georgia Cemetery Using Ancient DNA Analysis

Author(s): Andrew T. Ozga, Raúl Y. Tito, Brian M. Kemp, Hugh Matternes, Alexandra Obregon-Tito, Leslie Neal and Cecil M. Lewis Jr.

Source: Human Biology, Vol. 87, No. 2 (Spring 2015), pp. 109-121

Published by: Wayne State University Press

Stable URL: https://www.jstor.org/stable/10.13110/humanbiology.87.2.0109

JSTOR is a not-for-profit service that helps scholars, researchers, and students discover, use, and build upon a wide range of content in a trusted digital archive. We use information technology and tools to increase productivity and facilitate new forms of scholarship. For more information about JSTOR, please contact support@jstor.org.

Your use of the JSTOR archive indicates your acceptance of the Terms \& Conditions of Use, available at https://about.jstor.org/terms 


\title{
Origins of an Unmarked Georgia Cemetery Using Ancient DNA Analysis
}

\author{
Andrew T. Ozga, ${ }^{1}$ Raúl Y. Tito, ${ }^{1}$ Brian M. Kemp,${ }^{2}$ Hugh Matternes, ${ }^{3}$ \\ Alexandra Obregon-Tito, ${ }^{1}$ Leslie Neal, ${ }^{1}$ and Cecil M. Lewis Jr. ${ }^{1}$ *
}

\begin{abstract}
Determining the origins of those buried within undocumented cemeteries is of incredible importance to historical archaeologists and, in many cases, the nearby communities. In the case of Avondale Burial Place, a cemetery in Bibb County, Georgia, in use from 1820 to 1950, all written documentation of those interred within it has been lost. Osteological and archaeological evidence alone could not describe, with confidence, the ancestral origins of the 101 individuals buried there. In the present study, we used ancient DNA extraction methods in well-preserved skeletal fragments from 20 individuals buried in Avondale Burial Place to investigate the origins of the cemetery. Through examination of hypervariable region I (HVRl) in the mitochondrial genome (mtDNA), we determined haplotypes for all 20 of these individuals. Eighteen of these individuals belong to the L or U haplogroups, suggesting that Avondale Burial Place was most likely used primarily as a resting place for African Americans. After the surrounding Bibb County community expressed interest in investigating potential ancestral relationships to those within the cemetery, eight potential descendants provided saliva to obtain mtDNA HVR1 information. Three individuals from Avondale Burial Place matched three individuals with oral history ties to the cemetery. Using the online tool EMPOP, we calculated the likelihood of these exact matches occurring by chance alone $(<1 \%)$. The present findings exhibit the importance of genetic analysis of cemetery origins when archaeological and osteological data are inconclusive for estimating ancestry of anonymous historical individuals.
\end{abstract}

A vondale Burial Place was unearthed in 2008 during a road expansion in Bibb County, Georgia (Figure 1). New South Associates, Inc. (NSA), a private consulting firm specializing in cultural resource management and historical preservation, under contract with the Georgia Department of Transportation (GDOT), uncovered 101 human burials but no grave markers of any kind (Matternes et al. 2012). An initial challenge was determining what community was responsible for its construction and then linking the cemetery to the appropriate existing community. Historical reviews of communities present in southern Bibb County during the nineteenth and twentieth centuries were conducted by both the GDOT and NSA (Matternes et al. 2012).

The cemetery was not tied to any church, burial society, or other known bodies, nor did it seem to be

${ }^{1}$ University of Oklahoma, Norman, Oklahoma.

${ }^{2}$ Department of Anthropology and the School of Biological Sciences, Washington State University, Pullman, Washington.

${ }^{3}$ New South Associates, Inc., Stone Mountain, Georgia.

*Correspondence to: Cecil M. Lewis Jr., Laboratories of Molecular Anthropology and Microbiome Research, University of Oklahoma Stephenson Research and Technology Center, 101 David L Boren Blvd., Norman, OK 73019. E-mail: cmlewis@ou.edu.

KEY WORDS: GENETICS, AFRICAN AMERICAN CEMETERY, MITOCHONDRIAL HAPLOTYPES.

Human Biology, Spring 2015, v. 87, no. 2, pp. 109-121. Copyright @ 2015 Wayne State University Press, Detroit, Michigan 48201 
FIGURE 1. The location of Avondale Burial Place (noted as Project Area) within Bibb County, Georgia (from Matternes et al. 2012).

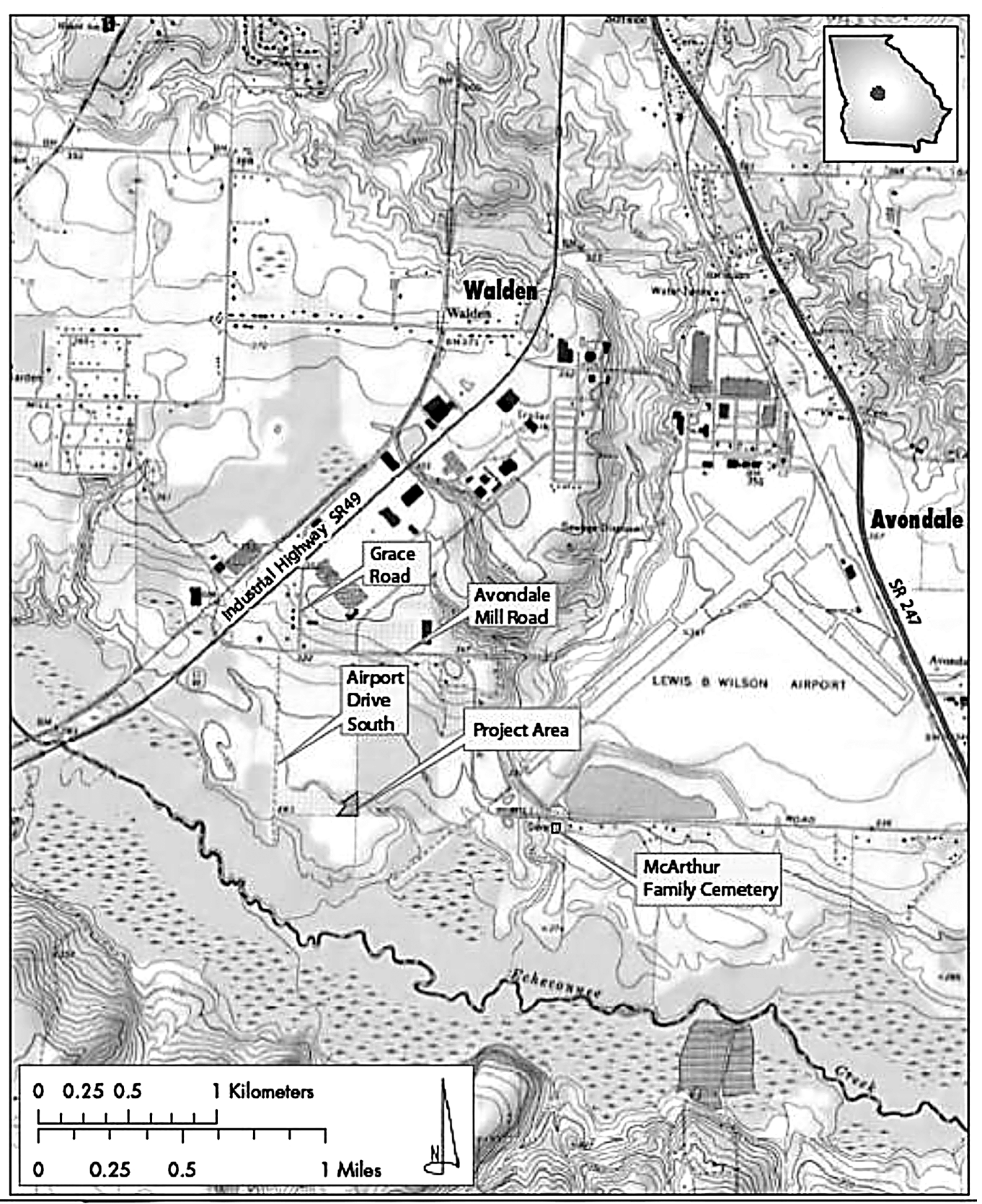

affiliated with any nineteenth- or twentieth-century landowners. A review of census records indicated that historically there had always been an African American presence in the area; however, the burial sites for many of these enclaves remained unknown. There were no maps or other records of the cemetery, and the lack of headstones hampered identification of specific decedents within the facility. The cemetery's position in the landscape, in an unusable corner of an agricultural field, adjacent to a wetlands area and away from habitation complexes, was similar to places commonly employed by African American communities (Martin 2010; Matternes and Richey 2014; Matternes and Smith 2014). While many nineteenth- and twentieth-century communities viewed cemeteries as means of social expression to the world at large, many African American communities perceived burial grounds as private, family-oriented facilities and placed them in discreet locations out of public view (Honerkamp and Crook 2012; Parsons 1923; Sloane 1991). GDOT and NSA investigations suggested that the burial ground 
was used by African American communities, but the near absence of historical records on the cemetery and highly ephemeral surface representation precluded making any definitive assertions about the cemetery's place in society.

Archaeological evidence suggests Avondale Burial Place was used by African Americans between 1820 and 1950, with the greatest number likely buried after the Civil War (Matternes et al. 2012). A concentration of graves was present in the central portion of the complex, with later period graves radiating outward. These simple, undecorated coffins with few to any accouterments suggested the Avondale Burial Place had its beginnings as a slave cemetery (Matternes et al. 2012). The graves were all oriented east-west, a common practice of both European-Christian and African-influenced burials. In the case of European Christians, burials are usually positioned in an east-west alignment with the head pointing west to allow the dead to face Jesus on his arrival; it is also symbolically attached to rebirth (Milbauer 1989). Early African burials in the United States are oriented in the same east-west direction but were intended to synchronize the dead with the structure of the universe (Genovese 1972; Pollitzer 1999). Other features, including vaulted graves and the use of hexagonal coffins, are shared among other African American cemeteries within the Southern United States (Matternes et al. 2012).

While grave markers had not survived, a light scatter of glass and white-bodied ceramic fragments was found on the cemetery's surface. Objects of this nature were commonly placed on the top of graves as a means of controlling the spirit and encouraging its passage to the world of the dead and were important decorations for Africans in the New
World (Evans et al. 1969; King 2010; Puckett 1926; Vlach 1990). Within the graves, pierced silver coins (Figure 2a) and seed bead necklaces were used as charms, designed to improve health and protect the wearer from magic (Capozzoli 1997; Crist et al. 2000; Davidson 2004; Evans et al. 1969; Puckett 1926; Stine et al. 2000). Beads are considered important personal artifacts within African American interments, but many within Avondale Burial Place were shattered by postdepositional forces prior to recovery, so the total amount could not be properly estimated. White, blue, black, and clear beads were identified from four interments and were between 1.5 and $2.0 \mathrm{~mm}$ in diameter (Figure 2b). Additionally, a sheet copper pendant depicting a crescent moon and a star may have reflected African-based Islamic ideas within the community (Figure 2c). Stars are commonly viewed as human souls, and the crescent moon symbolizes the beginning of a new life after crossing the Kalunga line into the world of the dead (Puckett 1926; Thompson 1984; Thompson and Cornett 1981). Gomez (2011) has demonstrated that pre-emancipation Muslim traditions were a part of African American heritage.

The burial of boots on top of one coffin related to African American traditions where footwear served as a means of protecting the dead from malevolent spirits (Davidson 2010). Personal items, including combs, dolls, tobacco pipes, purses, jewelry, and wedding bands, were placed with the dead to prevent the spirit from returning to their former homes searching for things they needed in the next world (Fenn 1985; Matternes et al. 2010; Roedinger 1981; Thompson and Cornett 1981; Vlach 1991). While not a uniquely African American feature, leaving coins on the eyes of the dead as a means of keeping the eyelids closed was a common phenomenon in
FIGURE 2. (A) Pierced silver coins (from F034, not used for genetic analysis) were among the charms used by African Americans to improve health and ward off deleterious magic. (B) Mixed color beads from one of the burials (F055, not used for genetic analysis) and a necklace clasp. These small glass artifacts, usually referred to as seed beads, have strong ties to West Africa. (c) A copper pendant from F007 depicting a star and crescent moon, also thought to be of West African tradition (Matternes et al. 2012).
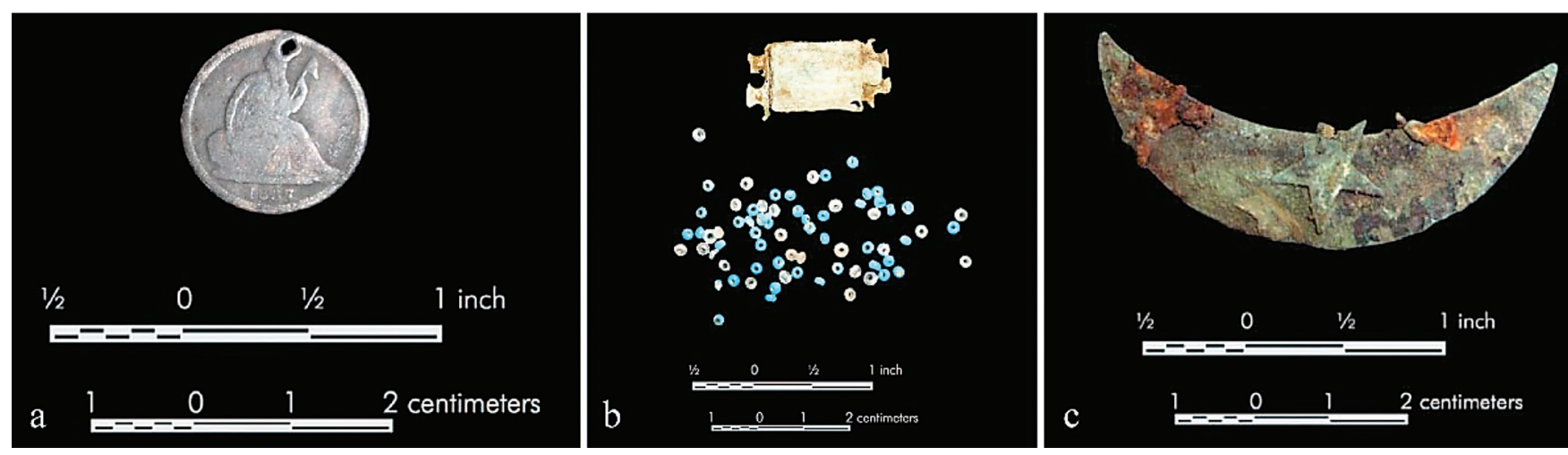

This content downloaded from 
African American graves (Crissman 1994; Matternes et al. 2010; Rose and Santeford 1985; Shogren et al. 1989). Overall, the material remains recovered during excavation suggest that the burial site was a mix of lowland/coastal African American funeral practices, as well as upland-like funeral traditions, found above the fall line, practiced by both African American and Anglo-American communities (Matternes et al. 2012).

The poor preservation of the 101 individuals recovered from Avondale Burial Place allowed for few conclusions regarding the lives of those buried there. Skeletal preservation was marginal, and definable skeletal features were found in 64\% of the 101 graves (Matternes et al. 2012). Bones of the legs and skull were the most represented, but unfortunately many indicators of age, sex, health, and ancestry found on delicate bones like the ribs, pubis, face, feet, and hands were least represented (Matternes et al. 2012). As a result, only 26 individuals could be identified for sex (16 females, 10 males). Age was estimated for 63 individuals using a combination of dental, cranial, skeleton developmental, vertebral, and os coxae markers (0-9.99 years, 35; 10-19.99, 5; 20-29.99, 3; 30-39.99, 7; $40-49.99,7 ;>50,6)$. Overall skeletal preservation was documented for each bone using a skeletal preservation battery that assigns a numerical scale ranging from 1.00 (bone in perfect condition) to 8.00 (completely decomposed, only organic stain present), with a score of 0 indicating a missing skeletal element. Scores at Avondale Burial Place ranged from 2.70 to 8.00 , with an average nonzero score of 6.004 for all 101 individuals (Matternes et al. 2012). A total of 10 crania from Avondale Burial Place were complete enough to be examined for ancestral affiliation using a variety of metric (Ousley and Jantz 1996) and nonmetric (Bass 1987; Rhine 1990; White and Folkens 2000) techniques (Table 1). Examinable cranial features were consistent with morphologies that would have been classified as African American by the local community. Faces were dominated by widened nasal apertures with well-developed nasal gutters that merged with moderately projecting zygomatics. These were features generally associated with people with ancestral ties to Africa (Bass 1987; Rhine 1990; White and Folkens 2000). Metric classification using discriminant function analysis also identified African populations as major contributors to expressed cranial dimensions. Aside from an os acromiale on the left scapula of F026, no pathological markers were present. Os acromiale is a genetic condition when the lateral end of the acromion fails to unite with the rest of the scapula (Mann and Hunt 2005), a feature more common among blacks than among whites (Sammarco 2000). Other health-related pathologies on skeletal remains within Avondale Burial Place were consistent with the African American health conditions from central Georgia at the time, including the presence of tendon ossification, hypercementosis, bowing of long bones due to rickets, and dental pathologies like malocclusions, hypoplasias, and periodontal disease (Matternes et al. 2012). The lack of conclusive archaeological and osteological evidence on the origins of the cemetery led to analysis using ancient DNA techniques at the University of Oklahoma.

mtDNA from skeletal remains has been successfully used in a number of studies to better understand population history and origin(s) (Adachi et al. 2009; Lee et al. 2009; Shook and Smith 2008; Stone and Stoneking 1998). Despite the poor preservation at Avondale Burial Place, it is still possible to recover portions of the mitochondrial genome using ancient DNA analysis techniques. In the present study, we estimated the maternal ancestry of those buried within Avondale Burial Place through an examination of mitochondrial hypervariable region I (mtDNA HVR1). Using this information it is possible to discern an individual's haplotype, a set of DNA polymorphisms that provide valuable insights into ancestral origin. Remains from 20 individuals, ranging in age from juvenile to adult, were selected for DNA extraction and mtDNA HVR1 amplification based on overall preservation (see Materials and Methods).

Contact was made with potential living descendants who had ancestral ties to the area's African American communities. Very few records about southern Bibb County's African Americans survive, and these descendants expressed considerable interest in what the cemetery could reveal about their heritage. Potential living descendants were also deeply concerned about whether the remains were truly those of their ancestors or belonged to other unknown individuals and communities. Within the African American community there is a strong movement to restore the historical, cultural, 
Table 1. Preservation and Nonmetric/Metric Assessment of the Individuals Used for Genetic Analysis

\begin{tabular}{|c|c|c|c|c|}
\hline \multirow[t]{2}{*}{ Burial } & \multirow{2}{*}{$\begin{array}{c}\text { Preservation } \\
\text { Score }\end{array}$} & \multicolumn{2}{|c|}{ Assessment $^{a}$} & \multirow[t]{2}{*}{ Notes (from Matternes et al. 2012) } \\
\hline & & Nonmetric & Metric & \\
\hline F001 & 4.60 & & & Shovel-shaped incisors \\
\hline F003 & 3.60 & & & \\
\hline F005 & 2.70 & & & \\
\hline F016 & 4.80 & AA & $\mathrm{AA} / \mathrm{C}$ & \\
\hline F017 & 4.80 & & & \\
\hline F018 & 5.20 & AA & NA & Skull broken, so very few measurements possible; results are suspect \\
\hline F023 & 4.90 & AA & NA & Skull broken, so very few measurements possible; results are suspect \\
\hline F024 & 5.10 & C/AA & C/AA & Individual may reflect Caucasian admixture \\
\hline F025 & 6.80 & & & \\
\hline F026 & 4.20 & AA & NA & Nasal infection may have influenced nasal dimensions \\
\hline F030 & 6.00 & & & \\
\hline F031 & 5.40 & AA & AA & \\
\hline F033 & 5.50 & & & \\
\hline F035 & 5.10 & AA & I & Very few observations possible \\
\hline F036 & 5.40 & & & \\
\hline F038 & 4.00 & C/AA & AA & Individual may reflect Caucasian admixture \\
\hline F039 & 5.80 & & & \\
\hline F051 & 6.50 & & & \\
\hline F096 & 4.40 & & & \\
\hline F100 & 7.80 & & & \\
\hline
\end{tabular}

${ }^{a}$ AA, African American; C, Caucasian; NA, Native American; I, intermediate.

and genealogical information in historical cemeteries like Avondale Burial Place (Carrier 2004). While historical, archaeological, and osteological information leaned strongly toward Avondale Burial Place representing an African American burial site, no definitive evidence to support this conclusion was found. Likewise, no evidence was found in the cemetery that specifically named decedents or linked the burial ground with the potential descendant community. Before accepting the cemetery as part of their own heritage, potential descendants desired further confirmation that these were truly the graves of their kinsmen.

A total of eight individuals with a history of slave ancestors in Bibb County provided saliva for mtDNA analysis. These individuals, living within and outside of Bibb County, are all hereby referred to as Bibb County participants. To establish potential maternal ancestry, we use the online tool EMPOP to determine if volunteers and interred individuals shared more haplotypes than expected by chance alone. Genetic data provided an independent and potentially more specific means of verifying what the archaeological and osteological data had to say about the ancestry of these individuals.

\section{Materials and Methods}

The University of Oklahoma Laboratories of Molecular Anthropology and Microbiome Research (LMAMR) were initially contacted by GDOT to extract DNA from the skeletal remains of individuals from Avondale Burial Place. Skeletal material from burials was selected for genetic analysis based on the following criteria: (a) would yield the greatest amount of DNA (ideally well-preserved teeth with an intact root), (b) could be linked to a single burial site with confidence (only a single individual within a site feature), and (c) could be recovered with minimal contamination from the excavation team (Matternes et al. 2012). A total of 19 teeth and a vertebra fragment, from 20 well-preserved skeletons, underwent DNA extraction at LMAMR (Table 2). Teeth or bone were pulverized using 
Table 2. Avondale Burial Place Skeletal Material used for Genetic Analysis

\begin{tabular}{llcc} 
Burial & Sample for DNA Testing & Skeletal Sex & Skeletal Age (years) \\
\hline F001 & Molar & $*$ & $9.5-14.5$ \\
F003 & Molar & F & $14-16$ \\
F005 & Molar & M & $35-40$ \\
F016 & Molar & M & $50-59$ \\
F017 & Molar & $*$ & $10-12$ \\
F018 & Premolar & F & $30-50$ \\
F023 & Vertebra fragment & F & $25-35$ \\
F024 & Molar & M & $45-49$ \\
F025 & Molar & $*$ & $12.5-17.5$ \\
F026 & Molar & M & $39-45$ \\
F030 & Incisor & $*$ & $4-8$ \\
F031 & Molar & M & $20-30$ \\
F033 & Molar & F & $50-59$ \\
F035 & Molar & M & $45-49$ \\
F036 & Molar & $*$ & $<7$ \\
F038 & Molar & M & $20-29$ \\
F039 & Molar & F & $38-52$ \\
F051 & Premolar & F & $<30$ \\
F096 & Molar & F & $35-45$ \\
F100 & Incisor & $*$ & Adult? \\
\hline
\end{tabular}

${ }^{\text {a}}$ Asterisks indicate sex unknown (lack of preservation, or child burial).

Table 3. HVR1 Primer Sequences

\begin{tabular}{llcc} 
Primer & DNA Sequence & Length (bp) & Ancient/Modern \\
\hline $15986 \mathrm{~F}$ & GCACCCAAAGCTAAGATTCTAATT & 168 & Ancient \\
$16153 \mathrm{R}$ & CAGGTGGTCAAGTATTTATGGT & & \\
$16106 \mathrm{~F}$ & GCCAGCCACCATGAATATTGT & 146 & Ancient \\
$16251 \mathrm{R}$ & GGAGTTGCAGTTGATGTGTGAT & & \\
$16190 \mathrm{~F}$ & CCCCATGCTTACAAGCAAGT & 166 & Ancient \\
$16355 \mathrm{R}$ & GGGATTGACTGTAATGTGCTATGT & & Ancient \\
$16232 \mathrm{~F}$ & CACACATCAACTGCAACT & 173 & \\
$16404 \mathrm{R}$ & GGTGGTCAAGGGACCCCTATCT & & Modern \\
$15971 \mathrm{~F}$ & CTTAACTCC ACCATTAGCA & 1082 & \\
$484 \mathrm{R}$ & TGAGATTAGTAGTATGGGAG & & \\
\hline
\end{tabular}

sterile tools by researchers equipped with sterile gowns, hair nets, and masks. DNA was extracted in an ISO 7 (class 10,000) HEPA-filtration, positivepressure clean room using previously established salting-out extraction methods (Tito et al. 2008, 2012). In brief, the salting-out protocol involved lysing the pulverized skeletal material overnight, followed by a proteinase $\mathrm{K}$ (Invitrogen) incubation and subsequent washing and eluting of the DNA
(Tito et al. 2008, 2012). Seven of these samples (F001, F017, F024, F035, F051, F096, F100) were replicated at Washington State University (WSU) using a modified protocol of Kemp et al. (2007) described by Moss et al. (2014).

The University of Oklahoma institute review board approved DNA collection from eight living individuals, recruited by NSA, who could trace their family history back at least two generations and whose oral family history suggested ancestors who were slaves in Bibb County. Saliva was collected from these individuals using the Oragene DNA collection kit (DNA Genotek, catalog no. OG-250), and DNA was extracted from each sample in accordance with the manufacturer's protocol.

At LMAMR, PCR amplifications were performed for the HVRl to sequence nucleotide positions 16011-16382, using the primers listed in Table 3. The mtDNA HVR1 PCR amplification was completed using previously established protocols (Grier et al. 2013; Tito et al. 2011). Agarose gels stained with ethidium bromide were used for visualization of HVR1 PCR products. Amplicons of the appropriate size were purified with exonuclease I and shrimp alkaline phosphatase and were sequenced in an ABI 3730XL capillary sequencer. For individuals with ambiguous haplotypes (F003, F005, F023, F024, F031, F033, F039, F051, F096), PCR products were cloned using TOPO TA Cloning Kits (Invitrogen) following the manufacturer's instructions and sequenced again using an $\mathrm{ABI}$ 3730XL capillary sequencer. At WSU, DNA extractions were tested for the presence of PCR inhibitors following the method of Grier et al. (2013). As none of the samples were demonstrated to contain sufficient levels of inhibition to preclude amplification, PCR amplification of four segments of the HVR1 and sequence preparation was conducted using previously established methods (Kemp et al. 2007).

The raw HVRI data were cleaned and compiled using Sequencher 4.10.1. The resulting modern and ancient HVRl sequences were compared with mthap (HVR1 region only) to determine the best haplogroup match (Lick 2014). Additionally, the online tool EMPOP (version 2.3, release 11; Parson and Dur 2007) was used to assess whether haplotype matches between Bibb County participants and Avondale Burial Place historical individuals are unlikely to have occurred by chance from a random sample of African Americans. EMPOP settings 
Table 4. Sample Haplotype Information

\begin{tabular}{|c|c|c|c|c|c|c|c|c|c|c|c|c|c|c|c|c|c|c|c|c|c|c|c|c|c|c|c|c|c|c|c|c|c|c|c|c|c|c|}
\hline$\exists$ & $\underset{\mathrm{g}}{\mathrm{g}}$ & 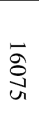 & $\begin{array}{l}\text { के } \\
\text { o }\end{array}$ & $\stackrel{\Xi}{\Xi}$ & $\frac{\vec{a}}{\sigma}$ & $\overrightarrow{\widehat{N}}$ & $\underset{\vec{a}}{\vec{f}}$ & $\underset{\vec{\infty}}{\vec{\infty}}$ & $\underset{\vec{a}}{\vec{\omega}}$ & $\vec{a}$ & $\underset{\vec{N}}{\vec{a}}$ & $\underset{\vec{\omega}}{\vec{\sigma}}$ & $\underset{\vec{\infty}}{\vec{a}}$ & $\vec{a}$ & $\vec{a}$ & $\overrightarrow{\vec{a}}$ & $\underset{\sigma}{\sigma}$ & 鸽 & $\underset{\infty}{\stackrel{\vec{N}}{\infty}}$ & $\begin{array}{l}\vec{\Omega} \\
\text { N } \\
\text { W }\end{array}$ & 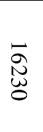 & $\underset{\stackrel{్}{心}}{\stackrel{心}{0}}$ & $\begin{array}{l}\overrightarrow{\tilde{J}} \\
\stackrel{8}{0}\end{array}$ & $\begin{array}{l}\vec{a} \\
\stackrel{\Delta}{+}\end{array}$ & $\underset{\mathrm{N}}{\overrightarrow{0}}$ & $\underset{\infty}{\vec{N}}$ & $\begin{array}{l}\overrightarrow{\hat{N}} \\
\stackrel{\infty}{\sigma}\end{array}$ & 空 & 空 & $\begin{array}{l}\vec{a} \\
\stackrel{0}{0} \\
+\end{array}$ & 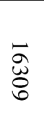 & $\underset{\stackrel{\vec{\omega}}{\Xi}}{ }$ & $\begin{array}{c}\vec{\Im} \\
\underset{\mathbf{J}}{\sigma}\end{array}$ & 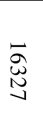 & $\begin{array}{l}\vec{a} \\
\vec{w} \\
u\end{array}$ & 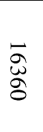 & 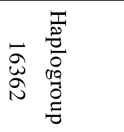 & $\begin{array}{l}3 \\
\frac{3}{0} \\
\overrightarrow{0} \\
0 \\
0\end{array}$ \\
\hline$\overline{\text { Ref }}$ & $\mathrm{T}$ & $\mathrm{T}$ & $\mathrm{T}$ & $\mathrm{C}$ & $\mathrm{T}$ & $\mathrm{G}$ & $\mathrm{G}$ & $\mathrm{C}$ & $\mathrm{A}$ & $\mathrm{C}$ & $\mathrm{T}$ & $\mathrm{A}$ & $\mathrm{C}$ & $\mathrm{C}$ & $\mathrm{C}$ & $\mathrm{C}$ & $\mathrm{T}$ & $\mathrm{T}$ & $\mathrm{C}$ & $\mathrm{C}$ & $\mathrm{A}$ & $\mathrm{C}$ & $\mathrm{C}$ & $\mathrm{C}$ & $\mathrm{C}$ & $\mathrm{C}$ & $\mathrm{C}$ & $\mathrm{C}$ & $\mathrm{A}$ & $\mathrm{C}$ & $\mathrm{A}$ & $\mathrm{T}$ & $\mathrm{C}$ & $\mathrm{C}$ & $\mathrm{C}$ & $\mathrm{C}$ & $\mathrm{T}$ & \\
\hline \multicolumn{39}{|c|}{ ANCIENT HAPLOTYPE INFORMATION } \\
\hline F001 & - & - & - & - & - & A & - & - & - & - & - & - & - & - & $\mathrm{T}$ & - & $\mathrm{C}$ & - & - & $\mathrm{T}$ & - & - & - & - & - & $\mathrm{T}$ & - & - & $\mathrm{G}$ & $\mathrm{T}$ & - & $\mathrm{C}$ & - & - & - & $\mathrm{T}$ & - L1c1 & $\wedge$ \\
\hline F003 & - & - & - & A & - & - & $\mathrm{A}$ & - & - & - & - & - & $\mathrm{T}$ & - & - & - & - & - & - & - & - & $\mathrm{T}$ & - & - & - & $\mathrm{T}$ & - & $\mathrm{T}$ & - & - & - & $\mathrm{C}$ & - & - & $\mathrm{T}$ & - & $-\mathrm{L} 2 \mathrm{e}$ & \\
\hline F005 & - & - & - & $\mathrm{T}$ & $\mathrm{C}$ & - & - & - & - & - & - & - & - & - & $\mathrm{T}$ & - & $\mathrm{C}$ & - & - & $\mathrm{T}$ & - & $\mathrm{T}$ & - & - & $\mathrm{T}$ & $\mathrm{T}$ & - & - & $\mathrm{G}$ & - & - & $\mathrm{C}$ & - & - & - & - & - L1b & \\
\hline F016 & - & $\mathrm{C}$ & - & - & - & - & - & - & - & - & - & - & - & - & - & - & - & $\mathrm{C}$ & - & $\mathrm{T}$ & - & - & - & - & - & - & - & $\mathrm{T}$ & - & - & - & $\mathrm{C}$ & - & - & - & - & - L3f1b & \\
\hline F017 & - & - & - & - & - & - & - & - & - & - & - & $\mathrm{C}$ & - & - & - & - & $\mathrm{C}$ & - & - & - & - & - & - & - & - & - & - & - & - & - & - & - & - & - & - & - & - $\mathrm{H} 2$ & \\
\hline F018 & - & - & $\mathrm{C}$ & - & - & - & - & - & - & - & - & - & - & - & - & - & - & - & - & $\mathrm{T}$ & - & - & - & - & - & $\mathrm{T}$ & - & - & - & $\mathrm{T}$ & G & - & - & - & - & - & - L2a1c & \\
\hline F023 & - & - & - & - & - & - & - & - & - & - & - & - & - & - & - & - & - & - & - & - & - & - & - & - & - & - & - & - & - & - & - & - & - & - & - & - & - $\mathrm{H} 2$ & \\
\hline F024 & - & - & - & - & - & - & - & - & - & - & - & - & - & - & - & - & - & - & - & $\mathrm{T}$ & - & - & - & - & - & - & - & - & - & - & G & - & - & $\mathrm{T}$ & - & - & - L3e1 & \\
\hline F025 & - & - & - & - & - & - & - & - & - & - & $\mathrm{C}$ & - & - & - & - & - & - & - & - & $\mathrm{T}$ & - & - & $\mathrm{T}$ & $\mathrm{T}$ & - & $\mathrm{T}$ & - & - & - & - & - & - & - & - & - & - & - U6a7b1 & \\
\hline F026 & - & - & - & - & - & - & - & - & - & - & - & - & - & - & - & - & - & - & - & $\mathrm{T}$ & - & - & - & - & - & $\mathrm{T}$ & - & - & - & $\mathrm{T}$ & G & - & - & - & - & - & - $\quad \mathrm{L} 2 \mathrm{a} 1$ & \\
\hline F030 & - & - & - & - & - & - & - & - & - & - & - & - & - & - & - & - & - & $\mathrm{C}$ & $\mathrm{T}$ & $\mathrm{T}$ & - & - & - & - & - & - & - & $\mathrm{T}$ & - & - & - & $\mathrm{C}$ & - & - & - & - & - L3f1b & \\
\hline F031 & - & - & - & - & - & - & - & - & - & - & - & - & - & - & - & - & $\mathrm{C}$ & - & - & $\mathrm{T}$ & - & - & - & - & - & $\mathrm{T}$ & - & - & - & $\mathrm{T}$ & G & - & - & - & - & - & - L2a1f & $\#$ \\
\hline F033 & - & - & - & - & - & - & - & - & - & - & - & - & - & - & - & - & $\mathrm{C}$ & - & - & $\mathrm{T}$ & - & - & - & - & - & $\mathrm{T}$ & - & - & - & $\mathrm{T}$ & G & - & - & - & - & - & - L2a1f & $\#$ \\
\hline F035 & - & - & - & - & - & - & - & - & - & - & - & - & - & - & - & - & - & - & - & $\mathrm{T}$ & - & - & - & - & - & $\mathrm{T}$ & - & - & - & $\mathrm{T}$ & G & - & - & - & - & - & - L2a1 & \\
\hline F036 & - & - & - & A & - & - & A & - & - & - & - & - & $\mathrm{T}$ & - & - & - & - & - & - & - & - & $\mathrm{T}$ & - & - & - & $\mathrm{T}$ & - & $\mathrm{T}$ & - & - & - & $\mathrm{C}$ & - & - & $\mathrm{T}$ & - & $-\mathrm{L} 2 \mathrm{e}$ & \\
\hline F038 & - & - & - & - & - & - & - & - & - & - & - & - & - & - & - & - & - & $\mathrm{C}$ & $\mathrm{T}$ & $\mathrm{T}$ & - & - & - & - & - & - & - & $\mathrm{T}$ & - & - & - & $\mathrm{C}$ & - & - & - & - & - L3f1b & \\
\hline F039 & - & - & - & - & - & - & - & - & G & - & - & - & - & - & - & - & - & - & - & 1 & - & - & - & - & - & - & - & - & - & - & - & - & $\mathrm{T}$ & - & - & - & - L3e2 & \\
\hline F051 & - & - & - & - & - & - & - & - & - & - & $\mathrm{C}$ & $\mathrm{C}$ & - & - & - & - & $\mathrm{C}$ & - & - & $\mathrm{T}$ & - & - & - & - & - & - & - & - & - & - & - & - & $\mathrm{T}$ & - & - & - & - L3e2b & \\
\hline F096 & - & - & - & - & - & A & - & $\mathrm{T}$ & - & $\mathrm{T}$ & $\mathrm{C}$ & - & - & - & $\mathrm{T}$ & G & $\mathrm{C}$ & - & - & $\mathrm{T}$ & $\mathrm{G}$ & - & - & - & - & - & - & - & - & - & - & $\mathrm{C}$ & $\mathrm{T}$ & - & - & - & C LOa1 & \\
\hline F100 & - & - & - & - & - & - & - & - & - & - & - & - & - & - & - & - & - & C & $\mathrm{T}$ & $\mathrm{T}$ & - & - & - & - & - & - & - & $\mathrm{T}$ & - & - & - & $\mathrm{C}$ & - & - & - & - & - L3f1b & \\
\hline A V002 & - & - & - & - & - & $\mathrm{A}$ & - & - & - & - & - & - & - & - & - & - & - & - & - & $\mathrm{T}$ & - & - & - & - & - & $\mathrm{T}$ & $\mathrm{T}$ & - & - & $\mathrm{T}$ & G & - & - & - & - & - & - L2a1 & \\
\hline AV003 & - & - & - & - & - & - & - & - & - & - & - & - & - & - & - & - & $\mathrm{C}$ & - & - & $\mathrm{T}$ & - & - & - & - & - & $\mathrm{T}$ & - & - & - & $\mathrm{T}$ & G & - & - & - & - & - & - L2a1 & \# \\
\hline AV006 & $\mathrm{C}$ & - & - & - & - & $\mathrm{A}$ & - & - & G & - & - & - & - & - & $\mathrm{T}$ & - & $\mathrm{C}$ & - & - & $\mathrm{T}$ & - & - & - & - & - & $\mathrm{T}$ & - & - & G & $\mathrm{T}$ & - & $\mathrm{C}$ & - & - & - & $\mathrm{T}$ & - L1c1 & \\
\hline AV007 & - & - & - & - & - & $\mathrm{A}$ & - & - & - & - & - & - & - & - & $\mathrm{T}$ & - & $\mathrm{C}$ & - & - & $\mathrm{T}$ & - & - & - & - & - & $\mathrm{T}$ & - & - & G & $\mathrm{T}$ & - & $\mathrm{C}$ & - & - & - & $\mathrm{T}$ & - L1c1 & $\wedge$ \\
\hline AV009 & - & - & - & - & - & - & - & - & - & - & - & - & - & - & - & - & - & - & - & $\mathrm{T}$ & - & - & - & - & - & - & - & - & - & - & - & - & - & - & - & - & $\mathrm{C} \quad \mathrm{D}$ & \\
\hline A V010 & - & - & - & - & - & $\mathrm{A}$ & - & - & - & - & - & - & - & - & $\mathrm{T}$ & - & $\mathrm{C}$ & - & - & $\mathrm{T}$ & - & - & - & - & - & $\mathrm{T}$ & - & - & G & $\mathrm{T}$ & - & $\mathrm{C}$ & - & - & - & $\mathrm{T}$ & - L1c1 & $\wedge$ \\
\hline AV013 & - & - & - & - & $\mathrm{C}$ & - & - & - & G & - & - & - & - & $\mathrm{T}$ & - & - & $\mathrm{C}$ & - & - & - & - & - & - & - & - & - & - & - & - & $\mathrm{T}$ & - & - & - & - & - & - & - $\mathrm{T} 1$ & \\
\hline AV014 & - & - & - & - & - & - & - & - & - & - & - & - & - & - & - & - & - & - & - & $\mathrm{T}$ & - & - & - & - & - & - & - & - & - & - & - & - & - & $\mathrm{T}$ & - & - & - L3e1 & \\
\hline
\end{tabular}

Sample haplotypes were designated using the best hit for HVR1 in the mthap database. For sample ID, F indicates historical samples, and AV indicates modern samples. Matches (\# and ^) denote an identical haplotype between historical and modern samples.

included nucleotide positions 16024-16365, disregarding length variants at position 16193. Bonferroni corrections (Dunn 1961) were used to conservatively estimate the probability of an exact HVRl match from Avondale Burial Place to a potential twenty-first-century descendant.

\section{Results}

The primary objective of this study was to determine the geographic origins of those interred within Avondale Burial Place. Eighteen of the 20 remains (90\%) exhibited HVR1 haplotypes that belong to either haplogroup L or haplogroup U, both of which indicate African ancestry (Table 4, Figure 3). The haplotypes of seven of these individuals were confirmed at WSU. The most abundant haplogroups were L2 $(n=7), \mathrm{L} 3(n=7)$, and L1 $(n=2)$. Two burials exhibited HVR1 haplotypes belonging to the $\mathrm{H} 2$ mitochondrial lineage. Overall, these results suggest that the Avondale Burial Place cemetery was used predominantly as an African American cemetery.

The secondary objective of this research was to use HVR1 to identify potential familial relationships 


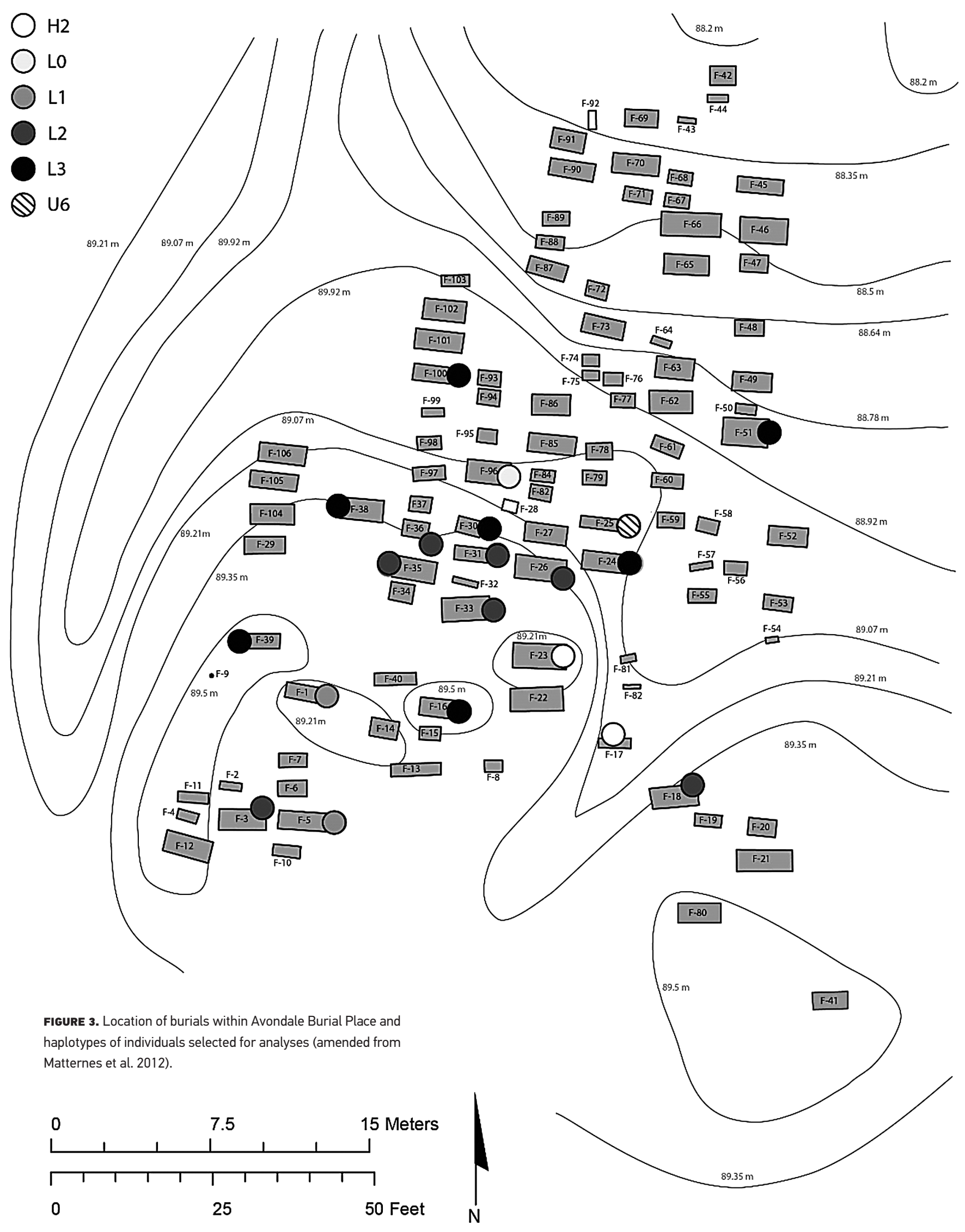


between Bibb County residents and those buried in Avondale Burial Place. The eight potential descendants possessed mtDNA HVRl sequences belonging to African ancestral haplogroups (L1, $n$ $=3 ; \mathrm{L} 2, n=2 ; \mathrm{L} 3, n=1)$, a Eurasian haplogroup T ( $n$ $=1)$, and an Asian haplogroup D $(n=1)$. Identical haplotype sequences between two sets of individuals from Avondale Burial Place and potential descendants (F001, F031, F033, AV003, AV007, and AV010) are denoted as matches in Table 4. These shared sequences were compared in the online tool EMPOP (version 2.3, release 11; Parson and Dur 2007). Using EMPOP, we set the parameters to search haplotypes with exact matches to the "USA" geographic affiliation with an "African" metapopulation. Table 5 shows the number of exact matches of each HVR1 mtDNA haplotype from Avondale Burial Place in relation to the total haplotypes in EMPOP $(n=34,617)$ prior to repeated number correction. Requiring exact haplotypes matches is a conservative criterion because recent mutation can be a confounding factor. Nevertheless, this conservative criterion finds putative relationships between historical and living participants. In this study, historical haplotypes were compared with eight unique modern haplotypes; therefore, a reasonable critical value after Bonferroni correction for each historical haplotype would be $0.05 / 8=$ 0.00625. The haplotype observed in F001, AV007, and AV010 has a probability less than this critical value (Table 6).

\section{Discussion}

\section{Origins of Avondale Burial Place}

The presence of individuals belonging to haplogroup L (and U) within Avondale Burial Place suggest that the cemetery was used for interment of African Americans. The results of this study confirmed that those interred within Avondale Burial Place shared genetic features associated with people of African origins. These people would have been identified locally as "colored" or "negro." These results would be consistent with African Americans living in rural Southern environments where physical and social factors limited access to the resources needed to maximize their quality of life. They lived during a period when the American death ritual emphasized a considerable outlay of
Table 5. Haplotype Matches in the EMPOP

\section{Database}

\begin{tabular}{ccc} 
Haplogroup & Burial & $\begin{array}{c}\text { Total Haplotypes } \\
\text { with Exact Matches }\end{array}$ \\
\hline H2 & F017 & 1 \\
H2 & F023 & 56 \\
L0 & F096 & 7 \\
L1 & F001 & 6 \\
L1 & F005 & 5 \\
L2 & F003 & 1 \\
L2 & F018 & 8 \\
L2 & F026 & 95 \\
L2 & F031 & 24 \\
L2 & F033 & 24 \\
L2 & F035 & 95 \\
L2 & F036 & 1 \\
L3 & F016 & 1 \\
L3 & F024 & 11 \\
L3 & F030 & 3 \\
L3 & F038 & 3 \\
L3 & F039 & 3 \\
L3 & F051 & 64 \\
L3 & F100 & 3 \\
U6 & F025 & 0 \\
\hline & & \\
\hline
\end{tabular}

Geographic affiliation, USA; metapopulation, African; total African haplotypes in America, 2,823; total haplotypes in EMPOP, 34,617. Table 6. Probability of Randomly Sampling
Haplotypes from 2,823 African American
Sequences Available in the EMPOP Database

\begin{tabular}{lcc} 
Haplotype Comparisons & $\begin{array}{c}\text { Observations } \\
\text { in EMPOP }\end{array}$ & Probability \\
\hline F001 AV007 AV010 & 6 & 0.0022 \\
F031 F033 AV003 & 24 & 0.0085 \\
\hline
\end{tabular}

capital for social display, but the use of homemade, locally made, and conservatively adorned coffins and attire implied that capital may not have been readily available for these rituals (Matternes et al. 2012; Sloane 1991). As noted in the introduction, inclusion of personal possessions, charms, and other objects reflected a continuation of traditions that likely had their origins in pre-emancipation African and African American communities. These genetic data in addition to the complementary archaeological, osteological, and oral history information helped validate, with some accuracy, 


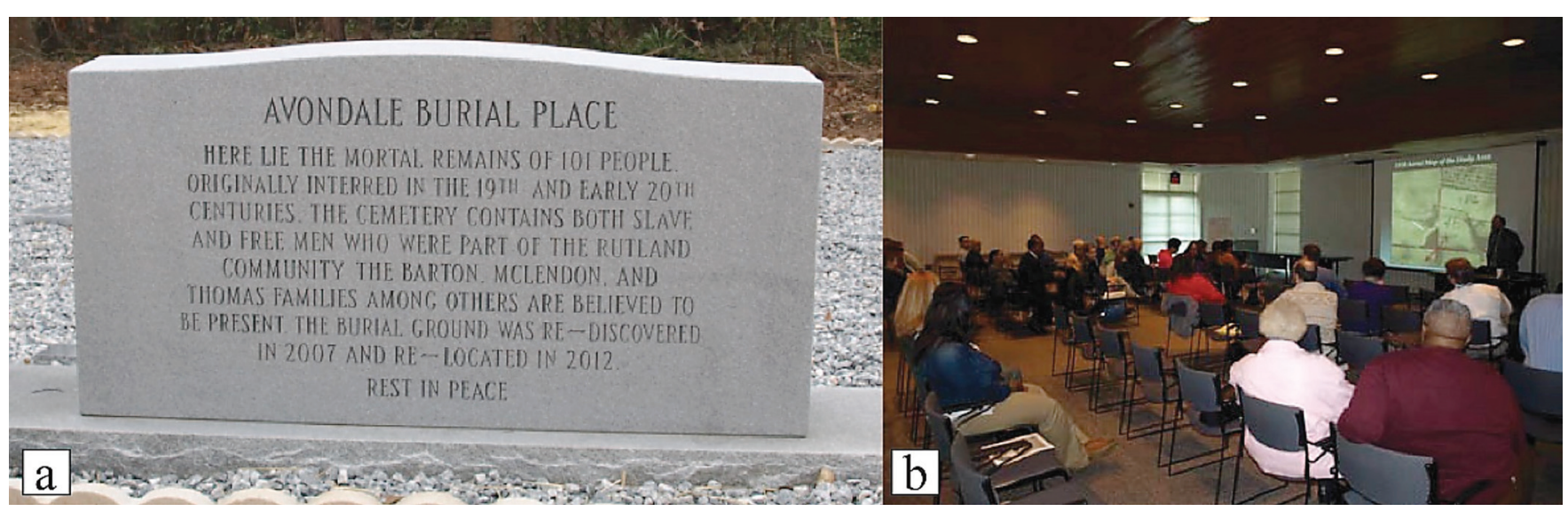

FIGURE 4. (A) Memorial stone placed beside the reinterment area, located next to Bethel AME church in Byron, Georgia. (B) Presentation of the findings at a public forum in Bibb County, Georgia (Matternes et al. 2012). the African American origins of the cemetery when little written information was available.

\section{Close Familial Relationships with Avondale Burial Place}

The genetic information from potential twentyfirst-century descendants was also compared with that of burials from Avondale Burial Place to assess familial relationships. Based on EMPOP sequences, the likelihood that two sets of exact matches, F001 with AV007 and AV010, and F031 and F033 with AV003, would occur by chance within this cemetery is less than $1 \%$. These historical and modern HVRl haplotype comparisons are compelling but should be used only for exploratory purposes. One limitation to this analysis is the use of only the mtDNA HVR1, but because of the overall state of preservation of the skeletal material, we did not attempt to obtain deeper autosomal data. Essentially, these data provide a proof of concept that with additional genome-level information, family relationships between these recently discovered unmarked cemeteries and living descendants can be estimated. Although only 20 burials were genetically examined in this study, this research gives a compelling reason for further evaluation of familial relationships in Avondale Burial Place using a deeper genome characterization. A future, genome-level characterization would allow for unprecedented outreach opportunities and facilitate family mapping within this cemetery to further evaluate potential descendants.

The HVR1 mtDNA information helped confirm that the living conditions reflected in Avondale Burial Place's skeletons were those of the African
American community. Degenerative conditions, including osteoarthritis, tendon ossification, and degenerative joint disease emphasized that many adults were engaged in physical labor that literally wore their bodies out (Matternes et al. 2012). They likely consumed a diet high in calories but low in nutrition, which lowered disease resistance and resulted in rickets, hypercementosis, and short stature and contributed to dental hypoplasias and periodontal disease (Matternes et al. 2012). This latter malady also reflected limited access to hygienic living environments. The harshness of these conditions was reflected in the sample of 63 individuals for whom skeletal age estimates could be obtained: fewer than 10 percent of the sample lived past age 50 , and over half died before reaching their 10th birthday (Matternes et al. 2012). Individuals interred in Avondale Burial Place represent a community living in considerable physical stress.

Oral histories from the descendant community placed their ancestors in southern Bibb County, and genetic results confirmed this part of the family history for three individuals. Abandonment of the cemetery occurred during the early twentieth century and corresponded with the general abandonment of the region by the descendant community (Matternes et al. 2012). Family and genealogical information outlined in Matternes et al. (2012) illustrate that movement of members of the community represented by Avondale Burial Place away from central Georgia in search of better opportunities was part of an African American exodus from the South generally referred to as the Great Migration (Wilkerson 2010).

The results of this study provided a sense of 
closure for members of the descendant community. Prior to discovery of the Avondale Burial Place, descendants had spent at least 20 years searching for the graves of their ancestors. Discovery offered hope and in three cases genetic analysis confirmed in the eyes of the descendant community that the bones in Avondale Burial Place included those of their ancestors. A missing chapter in family history had been restored, and descendants were secure in knowing that the bones they honored were those of their kinsmen. For the Bibb County African American community as a whole, important parts of their heritage, which had never been recorded or were lost by the passage of time, had now been recovered. The world represented by Avondale Burial Place was not a vignette from another unrelated community but was unquestionably that of their own people. Likewise, this study's results grounded the traditions, material culture, and quality of life reflected in the cemetery as features of the community's past. With this study, we utilized genetic evidence to expose the origins of Avondale Burial Place and potential ancestral relationships when osteological and archaeological evidence was inconclusive. When the remains were finally reinterred, family member Reverend Herman "Skip" Mason observed: "These must be very special people. We only get one funeral and these folks get two" (Matternes et al. 2012: 435) (Figure 4).

\section{ACKNOWLEDGMENTS}

The inquiries in this article were based on questions raised by members of the African American community, and we are thankful for their continued involvement and support. We are especially grateful that the Georgia Department of Transportation (GDOT) chose to fund this research. Walther Parson and Alexander Röck kindly provided EMPOP haplotype frequency data to assess the challenges of Bonferroni correction in this article. Special thanks to Julie Coco (NSA) for coordinating sample submission from potential descendants and to Valerie Davis, whose examination of the Avondale Burial Place's human remains provided important foundations for this study. The project was funded through the GDOT with some assistance from the Federal Highways Administration. The project was awarded to Adkins Global and subcontracted to New South Associates. The project was administered by GDOT's Office of Environmental Services Cultural Resource Program.
Received 20 April 2015; revision accepted for

publication 20 October 2015.

\section{LITERATURE CITED}

Adachi, N., K. Shinoda, K. Umetsu et al. 2009. Mitochondrial DNA analysis of Jomon skeletons from the Funadomari site, Hokkaido, and its implication for the origins of Native American. Am. J. Phys. Anthropol. 138:255-265.

Bass, W. M. 1987. Human Osteology: A Laboratory and Field Manual of the Human Skeleton. Columbia: Missouri Archaeological Society.

Capozzoli, M. M. 1997. A rip in the flesh, a tear into the soul: An ethnography of dissection in Georgia. In Bones in the Basement: Postmortem Racism in 19th Century Medical Training, R. L. Blakeley and J. Harrington, eds. Washington, DC: Smithsonian Institution Press, 313-339.

Carrier, T. 2004. Cemetery preservation: A growing concern, University of South Florida Africana Heritage Project, www.africanaheritage.com/cemetery.asp.

Crissman, J. K. 1994. Death and Dying in Central Appalachia. Urbana: University of Illinois Press.

Crist, T. A., W. R. Henry, J. W. Joseph et al. 2000. With Death Came Liberty: The Archaeology and History of the Sam Goode Cemetery, Mecklenburg County, Virginia. Stone Mountain, GA: New South Associates, Inc.

Davidson, J. 2004. Rituals captured in the context of time: Charm use in North Dallas Freedman's Town (1869-1907), Dallas, Texas. Hist. Archaeol. 38:22-54.

Davidson, J. 2010. Keeping the devil at bay: The shoe on the coffin lid and other grave charms in nineteenth and early twentieth century America. Int. J. Hist. Archaeol. 14:614-649.

Dunn, O. J. 1961. Multiple comparisons among means.J. Am. Stat. Assoc. 56:52-64.

Evans, D. K., D. S. Rice, and J. K. Partin. 1969. Parallels in West African, West Indian, and North Carolina folklore. N. C. Folkl. J. 17:77-84.

Fenn, E. 1985. Honoring the ancestors: Kongo-American graves in the American South. South. Expos. 8:42-47.

Genovese, E. 1972. Roll Jordan Roll: The World the Slaves Made. New York: Vintage Books.

Gomez, A. 2011. Africans, Culture, and Islam in the Low Country. In African American Life in the Georgia Low Country: The Atlantic World and the Gullah Geechee, P. Morgan, ed. Athens: University of Georgia Press, 103-130.

Grier, C., K. Flanigan, M. Winters et al. 2013. Using ancient DNA identification and osteometric measures of archaeological Pacific salmon vertebrae for reconstructing 
salmon fisheries and site seasonality at Dionisio Point, British Columbia. J. Archaeol. Sci. 40:544-555.

Honerkamp, N., and M. R. Crook Jr. 2012. Archaeology in a Geechee Graveyard. Southeast. Archaeol. 31:103-114.

Kemp, B. M., R. S. Malhi, J. McDonough et al. 2007. Genetic analysis of early Holocene skeletal remains from Alaska and its implications for the settlement of the Americas. Am.J. Phys. Anthropol. 132:605-621.

King, C. 2010. Separated by death and color: The African American cemetery of New Philadelphia, Illinois. Hist. Archaeol. 44:125-137.

Lee, E. J., L. M. Anderson, V. Dale et al. 2009. mtDNA origins of an enslaved labor force from the 18th century Schuyler Flatts Burial Ground in colonial Albany, NY: Africans, Native Americans, and Malagasy? J. Archaeol. Sci. 36:2805-2810.

Lick, J. 2014. mthap, data version 16.0, http://dna.jameslick. com/mthap/.

Mann, R. W., and D. R. Hunt. 2005. Photographic Regional Atlas of Bone Disease. Springfield, IL: Thomas.

Martin, K. 2010. Community and Place: A Study of Four African American Benevolent Societies and Their Cemeteries. Clemson, SC: Clemson University.

Matternes, H. B., V. Davis, J. Coco et al. 2012. Hold Your Light on Canaan's Shore: A Historical and Archaeological Investigation of the Avondale Burial Place (9BI164). Stone Mountain, GA: New South Associates.

Matternes, H. B., V. Davis, S. McIntyre et al. 2010. Shimmering Glimpses of the Spirit World: Archaeological Recovery of Two Historic Cemeteries (9CH875 and 9CH1168) at Hunter Army Airfield, Chatham County Georgia. South Mountain, GA: New South Associates.

Matternes, H. B., and S. Richey. 2014. I cry "I am" for all to hear me: The informal cemetery in central Georgia. In Natural Expressions of the African Diaspora, A. Ogundaran and P. Saunder, eds. Bloomington: University of Indiana Press, 258-279.

Matternes, H. B., and G. Smith. 2014. Preservation plan for the Dunwoody Cemetery. Stone Mountain, GA: Ceylon Cemetery Trust, Atkins Global, and Georgia Department of Transportation.

Milbauer, J. A. 1989. Southern folk traits in the cemeteries of northeastern Oklahoma. South. Folkl. 46:175-185.

Moss, M. L., K. G. Judd, and B. M. Kemp. 2014. Can salmonids (Oncorhynchus spp.) be identified to species using vertebral morphometrics? A test using ancient DNA from Coffman Cove, Alaska.J. Archaeol. Sci. 41:879-889.

Ousley, S. D., and R. L. Jantz. 1996. FORDIC 2.0: Personal computer forensic discriminant functions. Knoxville: University of Tennessee-Knoxville.
Parson, W., and A. Dur. 2007. EMPOP: A forensic mtDNA database. Forensic Sci. Int. Genet. 1:88-92.

Parsons, E. C. 1923. Folklore of the Sea Islands, South Carolina. Cambridge, MA: American Folklore Society.

Pollitzer, W. S. 1999. The Gullah People and Their African Heritage. Athens: University of Georgia Press.

Puckett, N. N. 1926. Folk Beliefs of the Southern Negroes. New York: Dover.

Rhine, S. 1990. Non-metric skull racing. In Skeletal Attribution of Race, G. W. Gill and S. Rhine, eds. Albuquerque: University of New Mexico, 9-20.

Roedinger, D. 1981. And die in Dixie: Funerals, death and Heaven in the slave community, 1700-1865. Mass. Rev. 22:163-183.

Rose, J. C., and L. G. Santeford. 1985. Burial descriptions. In Gone to a Better Land, J. C. Rose, ed. Fayetteville: Arkansas Archaeological Survey, 39-129.

Sammarco, V. J. 2000. Os acromiale: Frequency, anatomy and clinical implications. J. Bone Joint Surg. 82:394-400.

Shogren, M., K. R. Turner, and J. C. Perroni. 1989. Elko Switch Cemetery: An Archaeological Perspective. Tuscaloosa: University of Alabama, Office of Archaeological Research.

Shook, B. A., and D. G. Smith. 2008. Using ancient mtDNA to reconstruct the population history of northeastern North America. Am. J. Phys. Anthropol. 137:14-29.

Sloane, D. C. 1991. The Last Great Necessity: Cemeteries in American History. Baltimore, MD: Johns Hopkins University Press.

Stine, L. F., M. A. Cabak, and M. D. Groover. 2000. Blue beads as African American cultural symbols. In Approaches to Material Culture Research for Historical Archaeologists, 2nd ed., D. Brauner, ed. Tucson, AZ: Society for Historical Archaeology, 221-247.

Stone, A. C., and M. Stoneking. 1998. mtDNA analysis of a prehistoric Oneota population: Implications for the peopling of the New World. Am. J. Hum. Genet. 62:1153-1170.

Thompson, R. F. 1984. Flash of the Spirit: African-American Art and Philosophy. New York: Vintage Press.

Thompson, R. F., and J. Cornett. 1981. The Four Moments of the Sun: Kongo Art in Two Worlds. Washington, DC: National Gallery of Art.

Tito, R. Y., S. L. Belknap III, K. D. Sobolik et al. 2011. Brief communication: DNA from early Holocene American dog. Am. J. Phys. Anthropol. 145:653-657.

Tito, R. Y., D. Knights, J. Metcalf et al. 2012. Insights from characterizing extinct human gut microbiomes. PLoS One 7:e51146.

Tito, R. Y., S. Macmil, G. Wiley et al. 2008. Phylotyping and 
functional analysis of two ancient human microbiomes. PLoS One 3:e3703.

Vlach, J. M. 1990. Afro-American Tradition in Decorative Arts. Athens: University of Georgia Press.

Vlach, J. M. 1991. By the Work of Their Hands: Studies in African-American Folk Life. Charlottesville: University Press of Virginia.

White, T. D., and P. A. Folkens. 2000. Human Osteology. San Diego, CA: Academic Press.

Wilkerson, I. 2010. The Warmth of Other Suns. New York: Random House. 